\title{
Trivium
}

Revue franco-allemande de sciences humaines et sociales - Deutsch-französische Zeitschrift für Geistesund Sozialwissenschaften

15 | 2013

La science pense en plusieurs langues

\section{Moment, instant, occasion}

Françoise Balibar, Philippe Büttgen, Jean-Pierre Cléro, Jacques Collette et Barbara Cassin

\section{OpenEdition}

\section{Journals}

Édition électronique

URL : http://journals.openedition.org/trivium/4638

DOI : $10.4000 /$ trivium.4638

ISSN : 1963-1820

Éditeur

Les éditions de la Maison des sciences de l'Homme

\section{Référence électronique}

Françoise Balibar, Philippe Büttgen, Jean-Pierre Cléro, Jacques Collette et Barbara Cassin, « Moment, instant, occasion », Trivium [En ligne], 15 | 2013, mis en ligne le 09 décembre 2013, consulté le 08 septembre 2020. URL : http://journals.openedition.org/trivium/4638 ; DOI : https://doi.org/10.4000/ trivium. 4638

Ce document a été généré automatiquement le 8 septembre 2020

\section{(c) (i) (2)}

Les contenus des la revue Trivium sont mis à disposition selon les termes de la Licence Creative Commons Attribution - Pas d'Utilisation Commerciale - Pas de Modification 4.0 International. 


\title{
Moment, instant, occasion
}

\author{
Françoise Balibar, Philippe Büttgen, Jean-Pierre Cléro, Jacques Collette et
} Barbara Cassin

\section{NOTE DE L'ÉDITEUR}

Ce texte est une entrée de dictionnaire qui illustre de manière exemplaire et concrète l'un des problèmes majeurs que soulève la thématique générale de ce numéro de Trivium.

Nous remercions Mme. Barbara Cassin ainsi que les maisons d'éditions Le Seuil et Le Robert de nous avoir accordé l'autorisation de traduire ce texte pour le présent numéro.

Bei diesem Beitrag handelt es sich um einen Lexikoneintrag, der exemplarisch und ganz konkret eines der Grundprobleme der in diesem Heft zur Diskussion gestellten Thematik illustriert (s. Einleitung).

Wir danken Frau Barbara Cassin und den Verlagen Seuil und Le Robert für die freundliche Genehmigung, diesen Artikel in deutscher Übersetzung zu publizieren.

\begin{tabular}{|c|c|}
\hline gr. & 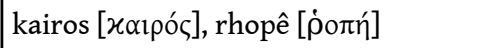 \\
\hline lat. & momentum \\
\hline all. & der Moment, das Moment, Augenblick \\
\hline angl. & momentum, moment, instant \\
\hline dan. & øjeblik \\
\hline it. & momento \\
\hline
\end{tabular}

1 > aiôn, aufheben, Dasein, destin, force, histoire, Jetztzeit, mot d'esprit, présent, temps

2 Moment a deux sens qui dérivent l'un de l'autre : un sens technique (mécanique) et un sens temporel. Mécanique : le momentum latin renvoie concrètement, via Archimède, à la petite quantité qui fait pencher la balance. Temporel : ce bougé détermine un avant 
et un après irréductibles l'un à l'autre. Cette irruption de temps dans l'espace est une clef pour comprendre le kairos grec, que l'on traduit, entre autres, par moment.

Les langues modernes se caractérisent par leur manière d'oublier le sens technique dans l'usage courant qui se focalise sur la détermination temporelle de petit laps de temps (cf. l'art. "Moment» dans L'Encyclopédie de Diderot et D’Alembert : «Un moment n'est pas long; un instant est encore plus court»). Parallèlement, elles spécifient de manière différente le sens technique.

4 L'allemand a en outre différencié par le genre le sens technique du sens temporel; Hegel fait du sens technique un usage spéculatif qui oblige à réorganiser la distinction d'avec le sens temporel. Le lexique philosophique des autres langues reprend par traduction l'usage hégélien («moment » comme instance, niveau de réalité).

\section{I. « Momentum » (lat.), « rhopê » (gr.) et leurs traductions}

5 Le sens technique du mot latin momentum est premier par rapport au sens de petit intervalle de temps. Momentum désigne alors une grandeur liée au mouvement. La redéfinition de la catégorie de mouvement opérée par Galilée et Newton conduit à établir dans les langues modernes une distinction linguistique, différente d'une langue à une autre, entre un sens dynamique et un sens statique confondus en latin.

6 Même dans son acception technique, momentum, qui est dérivé de movimentum (de movere, se déplacer), n'a pas un sens univoque. Cette polysémie reflète une difficulté rencontrée à partir du $\mathrm{XIII}^{\mathrm{e}}$ siècle dans la traduction du terme grec rhopê, terme utilisé au livre IV de la Physique d'Aristote (216a 13-20) et dans le Commentaire rédigé par Eutocios (VIe siècle) à partir du livre d'Archimède connu en français sous le titre Sur l'équilibre des figures planes (cf. Archimedis opera omnia cum commentariis Eutoccii, Teubner, 1915-1972, III, 264, 13-14).

7 Chez Aristote et chez Eutocios, rhopê désigne l'inclination qu'a un corps à se mouvoir selon le mouvement naturel avec une vitesse proportionnelle à son poids (ou à sa légèreté chez Aristote). Mais, dans l'ouvrage d'Archimède cité, rhopê a, via la considération de la balance, le sens de poids susceptible de faire pencher la balance d'un côté plutôt que de l'autre. Il s'agit toujours d'une inclination, mais celle-ci résulte alors de la combinaison du poids et de la distance au point d'appui du fléau de la balance. Certains traducteurs qui utilisent momentum pour désigner le premier sens de rhopê sont contraints d'avoir recours à un autre terme (par exemple pondus) lorsqu'ils veulent signifier le second sens. Cet usage est d'ailleurs loin d'être général puisque Vitruve, au livre X du De architectura (1486), parle de momentum comme de l'effet combiné du poids et de la distance. De plus, momentum sert également au Moyen Âge à traduire le terme grec to kinêma que l'on trouve au livre VI de la Physique d'Aristote (I, 232a 9,10, et 241a 4) et qui désigne alors un indivisible de mouvement déjà réalisé.

8 À la fin du XVI ${ }^{e}$ siècle, le sens technique de momentum est donc triple : (a) inclination naturelle au mouvement sous l'effet de la gravité (sens dynamique); (b) produit du poids par la distance (sens statique) ; (c) petite quantité de mouvement. Dans les trois acceptions, la référence au mouvement du fléau d'une balance lorsqu'il penche est implicite : momentum contient l'idée contradictoire d'un équilibre (statique) et de sa rupture (dynamique) sous l'effet d'une cause infinitésimale. 
On retrouve ces trois sens, mêlés et distribués (moyennant des distorsions propres à chaque langue) dans l'usage technique que fait chacune des langues modernes européennes des dérivés du mot latin momentum, à savoir: moment en français, momentum et moment en anglais, momento en italien et das Moment en allemand.

10 Moment, en français moderne, désigne le résultat d'une opération mathématique bien précise, celle qui consiste à construire le produit vectoriel du vecteur position d'un point matériel par un vecteur ayant ce point pour origine; on parle alors du moment de ce vecteur (par rapport à l'origine choisie pour repérer la position). La force newtonienne étant un vecteur appliqué au point matériel sur lequel elle agit, on peut définir par cette opération le moment d'une force. C'est donc le sens (b) qui est ici privilégié, ainsi qu'une conception mathématique des grandeurs mécaniques, mettant l'accent sur leur construction. Par analogie, la grandeur appelée en français moment cinétique, construite de la même façon à ceci près que la force est remplacée par la quantité de mouvement (définie comme le produit de la masse par la vitesse, grandeur vectorielle), devrait s'appeler le moment de la quantité de mouvement. Tel n'est pas le cas, mais l'adjectif cinétique ajouté à moment vise à rappeler ce lien avec la vitesse du mobile considéré et à cinématiser, sinon dynamiser, la notion de moment.

11 En anglais, il existe deux mots dérivés du sens technique de momentum: momentum, directement importé, et moment, traduction du terme latin. Momentum en anglais désigne ce qu'en français on appelle la "quantité de mouvement». Cet usage est postérieur à Newton qui parle, en anglais, de quantity of motion et, en latin, de quantitatis motus. Quant à moment, il désigne, comme en français, le produit vectoriel du vecteur position par un autre vecteur. C'est ainsi qu'on parle de «moment of a force » et qu'on peut trouver dans la littérature ancienne l'expression «moment of momentum » pour désigner ce qu'en français on appelle moment cinétique.

12 L'importation de momentum répond à un souci conceptuel: mettre l'accent sur la connotation dynamique que confère à la «quantité de mouvement » la seconde loi de Newton (encore appelée "principe fondamental de la dynamique »), laquelle prescrit que la variation temporelle de la quantité de mouvement d'un mobile est égale à la force appliquée sur ce dernier. L'anglais momentum évoque alors une impulsion et retient donc en partie le sens ( $a$ ) de son homonyme latin; tout en le modifiant, puisque le momentum anglais résulte de l'application d'une force extérieure et n'est pas inhérent au corps (du fait de sa gravité); de plus, le terme anglais introduit une nuance de type $(c)$, dans la mesure où la loi de Newton est une loi différentielle, donc portant sur des infiniment petits.

13 En somme, le français marque la différence entre deux concepts, l'un dynamique et l'autre statique, en se servant de deux mots nettement différents (quantité de mouvement et moment) et en mettant par là même l'accent sur le mode de construction des grandeurs physiques concernées, alors que l'anglais, qui utilise deux termes phonétiquement voisins, garde trace de la polysémie latine. On a affaire non seulement à deux conceptions du rapport entre mathématiques et physique, mais plus profondément à deux représentations intuitives différentes du mouvement : il n'est pas indifférent que le mouvement soit quantifié en français et impulsif en anglais. On verra une preuve de la difficulté qu'eut cette distinction conceptuelle à s'établir dans le fait qu'en français classique (notamment dans L'Encyclopédie), moment désigne parfois la "quantité de mouvement». L'article "Mechanique» reproduit même un curieux 
raisonnement visant à justifier, à propos de la balance, l'utilisation d'un même terme pour deux notions différentes.

En allemand, l'usage de das Moment est plus ou moins identique à celui de moment en français. Le moment d'une force est das Kraftmoment. Cependant, comme la quantité de mouvement se dit Impuls (après s'être appelée Bewegungsgrösse, quantité de mouvement), le moment cinétique est, de façon plus logique qu'en français, appelé Impulsmoment. Pourtant, das Moment a une connotation plus dynamique que moment en français; témoin l'expression Drehmoment, littéralement moment de rotation, pour désigner le moment cinétique.

L'usage en italien actuel du terme momento s'apparente beaucoup à celui que fait le français actuel du terme moment. Mais il faut signaler une acception de momento dont l'importance historique est fondamentale, celle que lui donne Galilée, entre 1593 et 1598, en établissant un lien, jusqu'alors impensable et qui n'est repris dans aucune langue, entre les sens $(a)$ et $(b)$. Cette coalescence de sens correspond à une tentative avortée de Galilée pour faire dériver la dynamique de la statique sans l'intermédiaire de la cinématique, sur la base d'un parallèle entre la balance et le plan incliné.

«Momento e la propensione di andare al basso, cagionata non tanto dalla gravità del mobile, quanto dalla disposizione che abbinno tra di loro i diversi corpi gravi [Le moment est la propension à aller vers le bas, provoquée non pas tant par la gravité que par la disposition qu'ont entre eux les corps graves] » (Les Méchaniques, II, $2^{\mathrm{e}}$ définition).

\section{II. « Kairos »}

Le mot grec kairos qui peut correspondre au français moment, entendu comme «bon moment ", « moment opportun », « occasion » (cf. le titre de Crébillon fils, La Nuit et le Moment), désigne une singularité non mathématisable. La rhétorique latine (Quintilien, III, 6,$26 ; \mathrm{V}, 10,43)$ distingue ainsi du tempus generale ou khronos, lié à l'histoire et susceptible de datation, le tempus speciale ou kairos, temps marqué qui ou bien se répète périodiquement (comme la bonne saison dans un cycle naturel ou le moment propice à un type d'action dans la vie des hommes), ou bien survient de manière imprévisible, et elle le rend aussi par «tempus per opportunitatem» (G. Fabii Laurentii Victorini explanationum in rhetoricam Ciceronis libri duo, Halm I, 21 p. 207, 40) ou occasio (ibid., I, 27, 40).

17 Ce qui fait la spécificité du mot grec et engendre son amplitude, c'est qu'il a à l'origine un sens spatial et désigne un point critique de coupure et d'ouverture, comme l'atteste l'adjectif kairios seul présent dans l'Iliade, qui s'applique au défaut d'une cuirasse, point d'articulation ou d'assemblage (IV, 185 ; XI, 439; VIII, 326), et à la suture osseuse d'un crâne (VIII, 84), tous lieux où un trait peut pénétrer de manière fatale et décider du destin. Ainsi Euripide parle-t-il d'un homme « frappé au kairos» (Andromaque, 1120). On comprendra peut-être par là comment en latin la « tempe » (tempus, oris) et le « temps » (tempus, oris), au même titre que le «temple» (templum), peuvent être mis en rapport avec temnô « couper» (cf. temenos « enclos, lieu consacré, autel»).

18 Selon l'hypothèse avancée par Onians, le mot usuel kairos et le terme technique kairos ne font qu'un, la différence d'accent marquant comme souvent la spécification sémantique. Kaîros appartient au vocabulaire du tissage et désigne la tresse régulatrice qui sépare les fils de chaîne, souvent couplée avec le dispositif qui maintient le sommet 
de l'ouvrage: elle détermine l'espacement entre fils pairs et impairs qui permet l'entrelacs entre chaîne et trame. De même, kairos au sens usuel renvoie à l'ouverture d'un discontinu dans un continuum, à la trouée du temps dans l'espace ou du temps temporel dans le temps spatialisé: moment de crise dans le vocabulaire médical, entrelacs ou concours de circonstances en politique et en histoire, il dit l'à propos (donc la [juste] mesure, la brièveté, le tact, la convenance) et l'occasion (donc l'avantage, le profit, le danger), tout moment décisif qu'il s'agit, de manière plus ou moins normative ou esthétique, de saisir au passage, voire par les cheveux comme en témoignent les figurations du kairos sous les traits d'un jeune homme chauve ou rasé par derrière, mais avec un long toupet par devant. C'est ainsi que, chez Pindare, le kairos caractérise les mots, à la fois bien décochés et bien tissés, qui touchent leur but (Néméennes, 1, 18 ; Pythiques, 1, $81 ; 9,78$ ).

19 L'attention prêtée au kairos définit un certain type de rhétorique, celle d'Alkidamas, d'Isocrate et des sophistes, et caractérise l'improvisation (gr. epi tôi kairôi lat. ex tempore) dont Gorgias fut pour Philostrate l'initiateur (Vitae Sophistarum, I, 482-483).

\section{III. « Der Moment » / « das Moment »}

20 L'allemand découple les significations simultanément présentes dans le latin momentum et les distribue non pas sur deux mots différents, mais sur deux genres d'un même mot, dont l'un est repris par le vocabulaire de la spéculation. Der Moment, au masculin, désigne un intervalle de temps plus ou moins long et das Moment, au neutre, a originellement le sens physique de "moment d'une force» (Momentum). Le lexique philosophique allemand, à partir de Kant, a produit une signification supplémentaire à partir de das Moment, celle de « cause, facteur, composante d'un tout considéré ou non dans son déroulement temporel ». À partir de là, il a forgé un terminus technicus de la spéculation, repris à ce titre par d'autres langues incluant le français. Cette création de la langue philosophique amène à poser deux questions.

21 La première est celle du rapport entre le sens mécanique et le sens spéculatif de das Moment. 
1 | « Moment » dans la Science de la logique

Le texte le plus topique est constitué par la remarque sur aufheben qui clôt le premier chapitre de la Science de la logique, soit le texte par excellence où la généralité du propos se nourrit de la particularité d'un idiome. Généralité par son environnement immédiat d'abord, puisqu'il conclut l'étape de la logique où sont intervenues les notions les plus abstraites, c'est-à-dire les moins déterminées, l'être, le néant et le devenir, et qu'il prend place au moment où le devenir prend fin et se conserve à la fois dans l'être-là (Dasein). Par sa visée propre ensuite, puisque Hegel, en explicitant le phénomène qui vient de se produire, l'Aufheben, entend traiter d' " un des concepts les plus importants de la philosophie ", dont le passage à l'être-là ne constitue qu'un exemple. Hegel présente ici une sorte de notice terminologique centrée sur le verbe aufheben (plutôt que sur le substantif Aufhebung) dans ses acceptions et usages divers. Si aufheben attire l'attention, c'est à cause d'un phénomène "réjouissant " que la "pensée spéculative» observe dans une langue particulière, l'allemand : le même verbe présente les deux significations opposées de «faire cesser, mettre un terme » et de " conserver, maintenir ». Pour pouvoir néanmoins penser autrement que « du point de vue lexical », il faut montrer comment « une langue en est arrivée à utiliser un seul et même mot pour deux déterminations opposées » en examinant ce qui se produit dans la chose même dont il est question. C'est à cette fin qu'est introduit le terme Moment :

«Etwas ist nur insofern aufgehoben, als es in die Einheit mit seinem Entgegengesetzten getreten ist; in dieser näheren Bestimmung als Reflektiertes kann es passend Moment genannt werden. Gewicht und Entfernung von einem Punkt heißen beim Hebel dessen mechanische Momente, um der Dieselbigkeit ihrer Wirkung willen bei aller Verschiedenheit eines Reelen, wie das Gewicht ist, und eines Ideellen, der bloßen räumlichen Bestimmung, der Linie

[Une chose n'est sursumée que dans la mesure où elle a accédé à l'unité avec son contraire ; selon cette détermination plus précise en tant qu'il s'agit d'un réfléchi, on peut de façon appropriée l'appeler moment. Poids et distance sont dans le cas du levier les moments mécaniques de ce dernier, en vertu de la similitude de leur action malgré toute la différence qu'il y a par ailleurs entre un réel, tel qu'est un poids, et un idéel, la pure détermination spatiale, la ligne]» (Hegel, Wissenschaft der Logik [Science de la logique], éd. de 1831, ch.1, Francfort, Suhrkamp, p. 114).

Il s'agit clairement d'une comparaison entre deux domaines distincts, celui de la spéculation où opère l'Aufheben et celui de la mécanique où l'on calcule des moments de forces. Moment y est présenté comme un emprunt, une «expression latine » dont se sert la "langue philosophique technique ». Dans l'Aufheben de la spéculation comme dans le Moment de la mécanique, les contraires, suppression et conservation, réel et idéel, opèrent de concert.

Un mot latin, momentum simplement germanisé en Moment, pour expliquer à des lecteurs allemands comment fonctionne un mot allemand, de la langue quotidienne qui plus est: l'opération est curieuse. Avec l'équivalence posée au début de la remarque entre das Aufgehobene (participe passé substantivé de aufheben) et das Ideelle mentionné dans le passage cité, elle doit suggérer l'existence d'un lien plus fort entre l'Aufheben et les Momente - celui-là même qu'établit la conclusion en évoquant « le sens et l'expression plus précise que reçoivent l'être et le néant à présent qu'ils sont des moments ». À ce moment, les Momente doivent être définis comme ce dont est fait le processus de l'Aufheben.

Il reste que ce processus même, pour ne faire ici référence qu'à la langue et à la mécanique, n'est jamais pensé tout à fait indépendamment du temps (cf. les verbes utilisés par Hegel: "mettre un terme" et "conserver»). C'est ce que montrent $a$ contrario les efforts de Marx après les Grundrisse pour ne plus penser en termes de «moments » des processus économiques qu'ils s'agit de faire échapper à l'eschatologie subreptice, à la correspondance entre cours du temps et mouvement du concept postulée par Hegel. 
Dans son traitement philosophique, das Moment retrouve donc la configuration du latin momentum et sa multiplicité de sens, mécanique et temporel. Le problème n'est alors pas tant que das Moment ait aussi un sens temporel : le français l'entend nécessairement dans moment - quoique la traduction se révèle malaisée dans certains contextes où le sens spéculatif prévaut malgré tout, témoin ce passage de Jaspers : «Der Augenblick hat in sich zum Beispiel ein [neutre] Moment der Angst [l'instant contient par exemple le moment de l'angoisse]» (K. Jaspers, Psychologie der Weltanschauungen, Berlin, Springer, 1925, p. 116.) Il est plutôt de savoir comment rendre différentiellement der Moment, maintenant qu'une bonne part du sens temporel s'est rabattue sur das Moment.

La seconde question porte donc sur la traduction de der Moment et du système de noms qui sert en allemand à exprimer le laps de temps dans ses durées inégales. Alors que le français ne dispose que du couple instant/moment, l'allemand peut jouer pour sa part avec trois termes : der Moment, das Moment avec ce qu'il comporte de sens temporel et der Augenblick. L' « opposition » (Gegensatz) du Zeitmoment et de l'Augenblick (Jaspers, op. cit., p. 114) obéit à une logique tout autre que l'opposition moment/instant. Augenblick seul se réserve le sens d' "instant vécu ", tandis que der [Zeit]moment peut dans certains cas désigner, non le moment, mais l'instant comme division objective, unité de mesure du temps (ibid., p. 111 : «der objektive Zeitmoment [l'instant objectif du temps]»; « ein beliebiger, willkürlich gewählter Moment [n'importe quel instant, choisi arbitrairement] »). Les difficultés de traduction propres à der/das Moment viennent donc aussi, par ricochet, révéler l'autonomie de la réflexion sur l'Augenblick. 
2 | Un hégélianisme de langue anglaise? « Moment » chez John Stuart Mill

Il est curieux de constater que John Stuart Mill, dans son Système de logique (publié en 1843, soit une dizaine d'années après le texte de Hegel cité ci-dessus), problématise la notion de moment à peu près dans le même sens que Hegel. Dans un chapitre qui traite des conditions d'un langage philosophique, il rappelle d'abord la signification dynamique de cette notion; puis, insistant particulièrement sur la vérité qu'elle enferme et qui porte sur la conservation de quelque chose d'inconnu (puisque le produit de la vitesse d'un corps par sa masse ne désigne, dans l'expérience, rien de réel), il lui fait jouer un rôle qui prend toute son importance dans l'usage des fictions tel qu'il le conçoit au Livre $\mathrm{V}$ : la notion, d'abord critiquée, est retournée dans une direction où elle est acceptée à d'autres conditions qu'auparavant. Tout le jeu de la théorie des fictions en usage chez les utilitaristes tient en effet dans la prise de conscience qu'un terme ne vise qu'en apparence quelque chose dans l'expérience, mais qu'il ne doit pas être rebuté pour autant, pourvu qu'on cesse d'être dupe de son illusoire transcendance, parce qu'il conserve un pouvoir indirect pour déterminer les choses.

«Il était déjà admis que, lorsque deux corps viennent à se choquer, le moment (the momentum) acquis par l'un est égal au moment perdu par l'autre. On jugeait nécessaire d'admettre cette proposition, non par le motif (décisif dans tant de cas) qu'elle était fermement établie dans l'opinion populaire, car la proposition en question n'avait jamais eu cours que parmi les savants ; mais on sentait qu'elle contenait une vérité. Une observation même superficielle des phénomènes ne laissait aucun doute que, dans la propagation du mouvement d'un corps à l'autre, il y avait quelque chose dont le second gagnait précisément ce que perdait le premier, et le mot Moment fut inventé pour exprimer cette chose inconnue (the word momentum has been invented to express this unknown something). La définition du moment impliquait donc la réponse à cette question: quelle est la chose dont un corps, quand il en met un autre en mouvement, perd une quantité égale à celle qu'il en communique ? Et lorsque l'expérience a montré que ce quelque chose était le produit de la vitesse du corps par sa masse ou quantité de matière, cette formule est devenue la définition du Moment (and when experiment had shown that this something was the product of the velocity of the body by its mass, or quantity of matter, this became the definition of momentum) » (J.S. Mill, Système de logique, trad. fr. L. Peisse, livre IV, ch. 4, § 4, p. 222-223 [A System of Logic, t. 2, p. 674]).

Cette analyse qui s'enracine dans la physique fait évidemment penser, étant donné son contexte logique et philosophique, à l'équivalent, dans une théorie des fictions, du Moment de l'Aufheben dans la Logique de Hegel. L'étrangeté de cette analogie tient à ce qu'elle s'effectue probablement à l'insu de Mill lui-même, en dépit de son intérêt fait de critique acerbe et d'admiration rentrée pour la philosophie allemande.

Bibliographie :

Mill John Stuart, A System of Logic Ratiocinative and Inductive, éd. J. M. Robson, Londres, Routledge, 1996; Système de logique déductive et inductive, trad. fr. de la $6^{\mathrm{e}}$ éd. angl. L. Peisse, Librairie philosophique de Ladrange, 1866, repr. Bruxelles, Mardaga, 1988.

Jean-Pierre Cléro

\section{IV. « Augenblick » / « instant »}

L'allemand représente l'instant non comme un point immobile sur une ligne (in-stans), mais comme un mouvement organique, le clin d'œil. L'Augen-blick allemand évoque à la fois la vitesse du regard et la lumière que celui-ci retient (cf. le poème de Schiller, Die Gunst des Augenblicks [La Faveur de l'instant]). Le mot signifie littéralement le « regard» et la "fermeture des yeux»; c'est le cillement de l'œil qui fixe son objet, puis par extension « la brève durée » d'une telle fermeture, qu'on s'accorde à considérer comme « indivisible» (Adelung, Grammatisch-kritisches Wörterbuch der Hochdeutschen Mundart, t. 1, Leipzig, $2^{\mathrm{e}}$ éd. 1793, sous art. « Augenblick », col. 561). 
métaphoricité particulière n'entraîne pas nécessairement de différence d'usage par rapport au français: le couple Moment/ Augenblick fonctionne comme le couple moment / instant, le deuxième terme se réservant à chaque fois la description d'un laps de temps si bref qu'il se dérobe à la mesure. Toutefois, alors que l'usage français impose le plus souvent d'ajouter une épithète quand instant désigne autre chose qu'une division objective du temps (voir par exemple G. Bachelard, L'Intuition de l'instant, Stock, 1932, p. 36: «un instant fécond»), c'est l'inverse qui se produit en allemand, où Augenblick seul désigne immédiatement l'instant vécu. Jaspers souligne que «le mot "Augenblick" caractérise quelque chose de tout à fait hétérogène dans ce qui reste identique sous les concepts formels du temps, à savoir le vide et le plein (das Erfüllte und Leere)». Ce qui conduit à la distinction terminologique suivante: "L'atome de temps (Zeitatom) n'est certes rien, mais l'instant (Augenblick) est tout» (K. Jaspers, op. cit., p. 108-117). Par là, Jaspers rassemble tout le processus au cours duquel l'Augenblick s'est trouvé doté d'une forte charge poétique et esthétique. La poésie développe particulièrement le thème de la part d'éternité contenue dans l'instant (cf. Goethe, Faust, I, V, 73), tandis qu'avec Lessing l'Augenblick devient un concept esthétique original, moment opportun distinct du kairos en ce qu'il cristallise une séquence temporelle, futur compris, au lieu de la rompre :

«La peinture ne peut, dans ses compositions où coexistent plusieurs temps, ne faire usage que d'un seul moment [Augenblick] et doit pour cela choisir le plus prégnant, à partir duquel ce qui précède et ce qui suit seront le plus compréhensibles » (Lessing, Laokoon, in Werke, Francfort, Deutsche Klassiker, 1967, vol. 2, p. 89).

La difficulté se précise lorsque l'ensemble de particularismes qu'on a mentionné se trouve comme revendiqué. Dans Sein und Zeit de Heidegger, le terme fait ses premières apparitions en deux paragraphes-clé marquant le passage à la temporalité originaire (\$§ 65 et 68). Augenblick sert alors à fixer les caractéristiques du " présent authentique » en tant que maintenu dans l'avenir et dans l'avoir-été (Heidegger, Sein und Zeit, Tübingen, Niemeyer, 1986, §. 68, p. 338 :

«In der Entschossenheit [...] wird [die Gegenwart] in der Zukunft und Gewesenheit gehalten [...] Die in der eigentlichen Zeitlichkeit gehaltene, mithin eigentliche Gegenwart nennen wir den Augenblick [Dans la résolution (...), le présent est tenu dans l'avenir et l'être-été. Le présent tenu dans la temporalité authentique, donc authentique, nous le nommons l'instant] », Être et Temps, trad. fr. E. Martineau, Authentica, 1985, p. 237).

Sur ce point, l'Augenblick est explicitement distingué du Jetzt, du maintenant de la temporalité dérivée qui comprend le temps comme un réceptacle, un milieu dans lequel les instants se succèdent. Instant, par le seul poids de son étymologie, apparaît alors comme une traduction malaisée pour Augenblick qui signale un présent qui luimême ne se tient pas dans le temps, un présent dans lequel rien ne se passe, puisque aussi bien il est seul à faire que le Dasein puisse s'ouvrir à un étant «dans un temps ». 
3 |“ Øjeblik” chez Kierkegaard angoisse

L'instant, qui est chez Kierkegaard l'objet de développements originaux dans le registre de l'existentialité, n'est assimilable à aucun des points du khronos: passé, présent, futur. Des deux termes danois, moment et øjeblik, le premier peut désigner, hors spéculation, les moments d'un tout ou d'un processus naturel ou historique. À noter cependant une occurrence non négligeable, puisque âme et corps sont dits deux «moments » d'une synthèse, l'esprit étant le troisième terme. La question du tiers posée quand les deux «moments » sont le temporel et l'éternel (t. 7, p. 185) aboutit précisément au concept d'øjeblik, le plus souvent préféré à moment pour connoter la dimension existentielle. Après les grands ouvrages où l'instant est une pièce maîtresse de l'analyse des stades esthétique et éthique, le concept est élaboré philosophiquement dans deux livres parus en 1844 : Miettes philosophiques et Le Concept d'angoisse, notamment au chapitre 3.

Sans l'instant, venue de Dieu lui-même dans le temps, «tout serait resté socratique » (t. 7, p. 53), le paradoxe aurait été manqué, en lequel temps et éternité se touchent. Ou encore, tout serait resté aux mains de «la négation, du passage, de la médiation, ces trois agents masqués, suspects et secrets qui [dans la Logique hégélienne] mettent tout en mouvement» (t. 7, p. 181). L'impulsion chrétienne de la réflexion conduit Kierkegaard à s'appuyer a contrario sur de solides piliers philosophiques (Socrate, l'exaiphnês platonicien, la philosophie idéaliste de la religion) pour donner, au prix « d'un malentendu productif» (W. Beierwaltes, « Exaiphnès... », p. 282), toute son extension au concept d'instant.

L'instant est un terme qui fait image : «Atome et clin d'œil » (t. 7, p. 187 : Paul, Première Épître aux Corinthiens, 15,52), il désigne la fin du temps tout en exprimant l'éternité. Comment interpréter cette « première tentative de suspendre le temps » ? Pour les Grecs, l'éternité est le passé auquel on n'accède qu'à reculons. Pour le judaïsme, l'histoire et l'avenir deviennent décisifs. Mais c'est le christianisme qui fait apparaître à la fois la différence qualitative absolue et le contact du temps et de l'éternité. L'avenir, loin d'être conçu comme résultat du passé, est « un tout dont le passé fait partie » (comparer avec Merleau-Ponty, Phénoménologie de la perception, Gallimard, 1945, p. 471). Mais il faut pour cela que l'instant soit posé concrètement, de sorte qu'apparaisse cette « ambiguïté en laquelle le temps intercepte [déchire: afskcere] l'éternité et l'éternité pénètre le temps » (t. 7, p. 188). L'instant, "plénitude du temps» (Paul, Épître aux Galates, 4,4, cité t.7, p.18, p. 189), «éternisation de l'historique et historisation de l'éternel» (t. 7, p. 58), signifie que l'éternel est « l'avenir qui fait retour comme le passé » (ibid. et t. 15, p. 92).

Comme Leucippe pour l'espace, Platon a posé la question du mouvement dans le temps. Son mérite est d'avoir ainsi découvert l'exaiphnês et sa soudaineté. Toutefois, son approche " métaphysique » ne peut qu'en faire une «muette abstraction atomique» (t. 7, p. 183). Tout en rendant justice aux Grecs (t. 5, p. 20), il convient de mieux cerner cette « chose étrange » (atopon, ce sans-lieu, ce pur entre-deux (mellem), intervalle entre mouvement et repos, ce passage kat'exokhên, « qui n'est dans aucun temps ». Il ne peut s'agir de ce qui « se passe dans le dos de la conscience » (Lectures, p. 304, 321). Extrait du contexte physique et métaphysique, transféré, sur la base du donné « dogmatique », dans le champ de l'existentialité, ce passage relève de la possibilité (ibid., p. 300). Il conditionne le jeu des catégories de saut, de décision, de répétition, de contemporanéité, là où travaillent l'instant opposé à la réminiscence, la disjonction opposée à la médiation.

Ainsi compris, l'øjeblik est, en des sens opposés, au centre des analyses de la foi et de l'angoisse, où il appert que le primat de l'avenir et le vertige de la liberté donnent toute son envergure à la dimension du possible, du pur entre-deux comme pouvoir. Comme « l'Un qui à la fois est et n'est pas », l'instant de « l'angoissante possibilité de pouvoir [...] forme supérieure de non-savoir [...], en un sens plus haut, est et n'est pas» (t. 7, p. 146,183). L'instant est à la fois temporel (passage) et " hors du temps».

La conception du temps étant chose décisive dans la détermination des stades de l'existence (esthétique, éthique, religieux), le concept d'instant sera l'objet de trois variations originales rythmées par une potentialisation croissante.

L'instant esthétique, le beau moment «poétique » est « l'instant éternel de la jouissance » (t. 2, p. 272). Éternel, car tout souci des contingences extérieures étant annulé, « il est tout » (t. 3, p. 401 ;

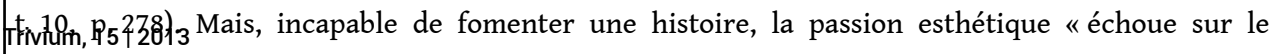
temps » (t. 10, p. $234 s q$.$) .$

"L'esthétique est en l'homme ce par quoi il est immédiatement ce qu'il est ; l'éthique est ce par quoi il devient ce qu'il devient» (t. 4, p. 162). Les modèles de ce devenir sont l'amour conjugal et l'action sociale, qui impliquent durée, continuité et histoire. C'est par l'instant du choix résolu de 
Dès lors se repose le problème de la métaphoricité propre à Augenblick. L'expression adverbiale en un clin d'œil fournit un équivalent valable, mais qui ne peut en tout état de cause être substitué systématiquement à un nom. La remarque d'Adelung, selon laquelle Augenblick doit s'entendre au sens figuré sans que jamais ou presque un sens propre ne soit en usage, peut être prise dans tout son sens : par rapport à Augenblick, instant, en ne rendant pas la métaphore, désigne une compréhension différente du temps et clin d'œil rend la métaphore, mais ne dit pas le temps.

INDEX

Schlüsselwörter : Wörterbuch, Übersetzung, Philosophie

Mots-clés : dictionnaire, traduction, philosophie 